\title{
Hipparcos Positions of B/Be Stars on the HR Diagram
}

\author{
Danielle Briot \\ Noël Robichon \\ DASGAL/UMR 8633,Observatoire de Paris-Meudon
}

\begin{abstract}
.
Absolute magnitudes of Be and B stars are computed for each spectral type and luminosity class V and IV, using the Hipparcos parallax measurements. Some simulations have been carried out in order to estimate the effects which could bias the mean absolute magnitude calcular tions. As a result, only stars with $\sigma_{\pi} / \pi<15 \%$ have been used. A first result is that B stars are fainter than previous estimations by about 0.5 magnitude on average. We then observe that on average Be stars are brighter than B stars of the same spectral type and this over-luminosity increases with the spectral type. A possible interpretation is proposed based on the fact that the rotational velocity of the late Be stars is near the critical rotational velocity.
\end{abstract}

\section{Introduction: the situation before Hipparcos}

The location of Be stars in the HR Diagram is a key to understand the Be process because it gives information about the evolutionary path, the luminosity of the central star and characteristics of the circumstellar envelope. Since Merrill (1933), many studies have investigated the absolute magnitudes of $B e$ stars compared to those of B stars (see references in Zorec and Briot 1991). However, $\mathrm{B}$ stars, and moreover Be stars are scarce in the solar neighbourhood. This implies that only very few or inaccurate ground-based parallax measurements are available for $\mathrm{B}$ stars and practically none for Be stars. Before the Hipparcos mission, absolute magnitudes of Be stars were only determined by various indirect methods: membership of binaries, open clusters or associations, or by statistical means based on their spatial and kinematical distributions. Because of the poor quality and the small size of the samples studied, the results obtained were contradictory. There was nevertheless a consensus of considering Be stars brighter (about 0.5 up to $1 \mathrm{mag}$ ) than B stars without emission.

The advent of the Hipparcos satellite changed the situation. Hipparcos followed during 37 months 118,218 stars and provided a final catalogue of positions, proper motions and parallaxes with a median precision of 1 mas (ESA 1997). 


\section{The samples}

The samples of B and Be stars used in the present study are built using the whole Hipparcos Catalogue (contrary to a preliminary study, Briot et al. 1997, which used preselected stars). In order to reduce evolutionary effects, we restrict to B and Be stars of luminosity classes V and IV. Known double stars which could bias the absolute magnitudes have also been excluded. The Hipparcos spectral types of Be stars have been revised using the BCD spectral types (Zorec \& Briot $1991)$ or the spectral types given in Slettebak (1982).

The Hipparcos catalogue contains 18,377 stars with spectral type in the range O8.5 - A1.5. Among them, 7,184 stars have a luminosity class V or IV and 6,061 out of them correspond to a single component observed photometrically (field H59 empty or field H59="C" and field H36 non equal to * or -). Among these stars, 323 are in the Jaschek-Egret Catalogue of Be stars (1982) and 21 have been quoted as Be stars in the literature since the publication of the JaschekEgret Catalogue. Nine other new Be stars without spectral type in the Hipparcos Catalogue have also been included to the Be sample. The final samples are then composed of $353 \mathrm{Be}$ stars and 5,718 B stars without emission. The number of $\mathrm{B}$ and $\mathrm{Be}$ stars for each spectral type is shown in Figure 1. It varies from $32 \mathrm{~B} 0$ stars to more than $1,000 \mathrm{~A} 0$ stars and from $8 \mathrm{Be} 0$ stars to $7 \mathrm{~A} 0 \mathrm{e}$ stars with a peak of 77 B2e stars.

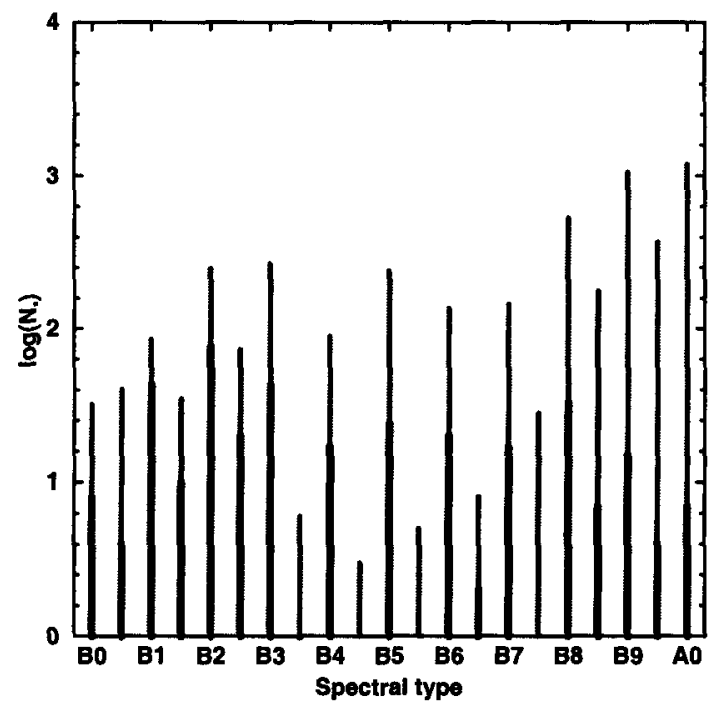

Figure 1. Number of B (thin lines) and Be (thick lines) stars vs Spectral type

The spectral type was preferred to the $(B-V)$ index as a temperature indicator, because of the intrinsic reddening of Be stars and the difficulty in estimating it. The interstellar absorption $A_{V}$ is computed using the tridimensional model of Arenou et al. (1992), where the colour excess distribution is 
modelled as a function of galactic latitude, longitude and distance. It is valid for stellar distances smaller than about $1 \mathrm{kpc}$ from the Sun.

Absolute magnitudes versus spectral types for each $B$ and Be star of the sample (with $\pi>0$ ), as well as the linear regressions weighted by the individual absolute magnitude errors, are displayed in Figure 2.

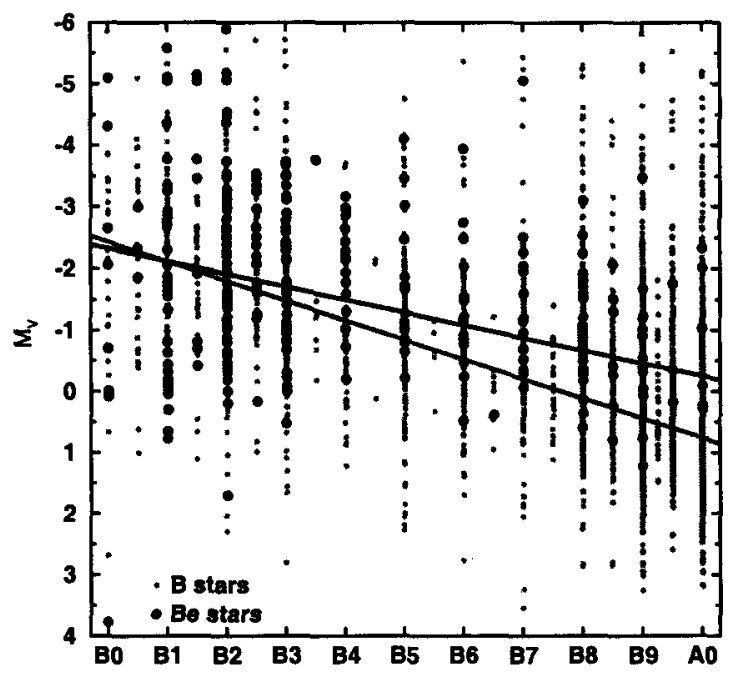

Figure 2. HR diagram of $\mathrm{B}$ and Be stars of the sample with $\pi>0$. The lines indicate linear regressions weighted by the individual errors on the absolute magnitudes.

\section{Detection and elimination of possible biases}

\subsection{Main biases in the determination of mean absolute magnitudes of a sample}

Some systematic errors can be introduced in the determination of the absolute magnitudes of a star sample. The most well-known ones, the Malmquist bias (1936) and the Lutz \& Kelker bias (1973), occur when truncating the sample in apparent magnitude or observed parallax.

The Malmquist bias (1936) appears when using a sample of stars with an intrinsic absolute magnitude dispersion and selected on their apparent magnitudes. In that case, there is an over-estimation of the mean luminosity of the sample stars because stars of brighter absolute magnitudes are preferably selected. The correction may vary depending on the absolute magnitude distribution and the spatial distribution of stars in the sample. Furthermore, if the apparent magnitude truncation is not clean-cut, the effect is difficult to model.

Another bias was described by Lutz \& Kelker (1973). This effect occurs for samples truncated according to their observed parallaxes. As the number of 
stars increases with the true distance, the observed parallax of a star is more likely larger than the true parallax. Then, the Lutz \& Kelker bias involves an under-estimation of the mean luminosity of the sample stars for small values of $\sigma_{\pi} / \pi$. For large values of $\sigma_{\pi} / \pi$, the bias inverses and involves an overestimation of the luminosity. It is due to the non-linear relationship between the absolute magnitude and the parallax of a star. This is also due to the fact that stars with negative parallaxes must be excluded to compute the absolute magnitude. Finally, this bias also strongly depends on the truncation on apparent magnitude which changes with the proportion of stars having small true parallaxes.

Arenou \& Luri (1999) reviewed all the biases occurring when trying to compute mean parameters on a star sample with observed parameters affected by random errors and selection effects and proposed statistical approaches to avoid or correct them.

\subsection{Estimation of the biases by simulations}

We can estimate the biases by making some simulations. We use a uniform distribution of stars in the Galactic plane and an exponential distribution in $\mathrm{z}$ with a scale height of $200 \mathrm{pc}$. The standard deviation $\sigma_{\pi}$ is distributed as in the Hipparcos catalogue as a function of $(\alpha, \delta)$ and $V$, the number of observations for each star depending of the scanning law of the satellite. $M_{V}$ and $\sigma_{M_{V}}$ are fixed. The cut-off in apparent magnitude is $V=9$. Figure 3 shows $M_{V}$ as a function of $\sigma_{\pi} / \pi$ obtained from the simulation for various fixed values of $M_{V}$ between -2 and 1 and $\sigma_{M_{V}}=0.7$. We can see that the biases depend of the mean absolute magnitude of the sample. For $M_{V}=-2$, there is first an under-estimation (Lutz \& Kelker bias) of the magnitude which reaches $0.8 \mathrm{mag}$ at $\sigma_{\pi} / \pi=0.4$ and then a decrease (mainly due to the Lutz \& Kelker bias but increased by the Malmquist bias) such that the bias is $-0.4 \mathrm{mag}$ at $\sigma_{\pi} / \pi=1$. For $M_{V}=1$, the Lutz \& Kelker bias is quite immediately overluminous and reaches 2.5 mag at $\sigma_{\pi} / \pi=1$. Figure 4 shows the same data as Figure 3, with an expanded abscissa scale. One can see that for $\sigma_{\pi} / \pi \leq 15 \%$, no bias larger than 0.1 mag is present when computing the mean absolute magnitudes.

Actually, the most important bias is due to the apparent magnitude truncation of the sample. As the apparent magnitude truncation of Hipparcos is not clear cut, the effect is very difficult to model and hence correct. The simulations we have done are close enough to the reality to estimate the order of magnitudes of the biases but not enough to compute accurate corrections to apply to the data. For that reason, we will consider only stars with $\sigma_{\pi} / \pi \leq 15 \%$ for which the mean absolute magnitudes are not noticeably biased. As compensation, of course, only few stars of each spectral type remain in the final samples, especially for very early-type stars.

\section{Results - Absolute magnitudes for $B$ and Be stars versus spectral type.}

The individual absolute magnitudes $M_{V}$ of $\mathrm{B}$ and Be stars with $\sigma_{\pi} / \pi \leq 15 \%$ are plotted as a function of spectral type on Figure 5. The regression lines, computed by taking into account the errors, are very similar to those of Figure 2 . This does not mean that the absolute magnitudes computed using the whole samples 


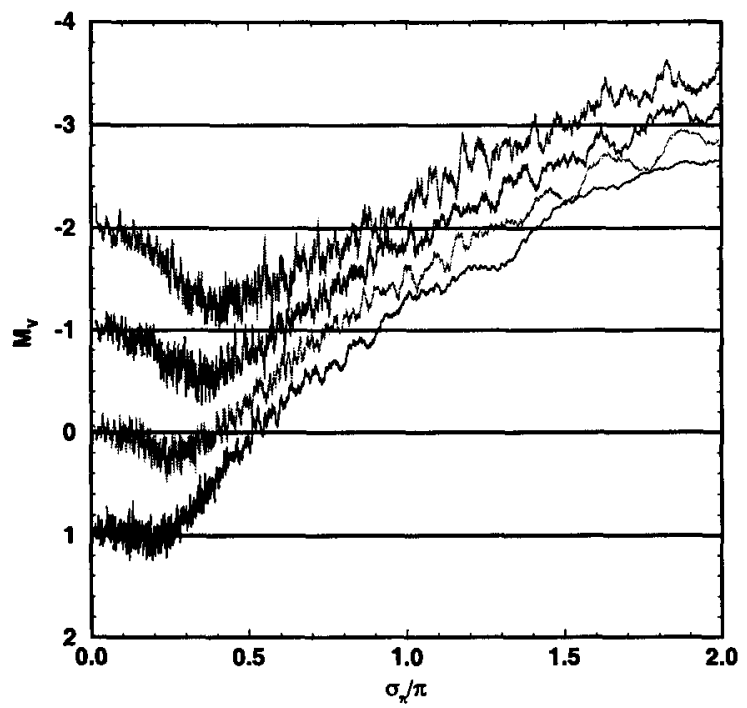

Figure 3. $M_{V}$ versus $\sigma_{\pi} / \pi$ from the simulation samples $\left(\sigma_{M_{V}}=0.7\right.$, $M_{V}=-2,-1,0$ and 1$)$

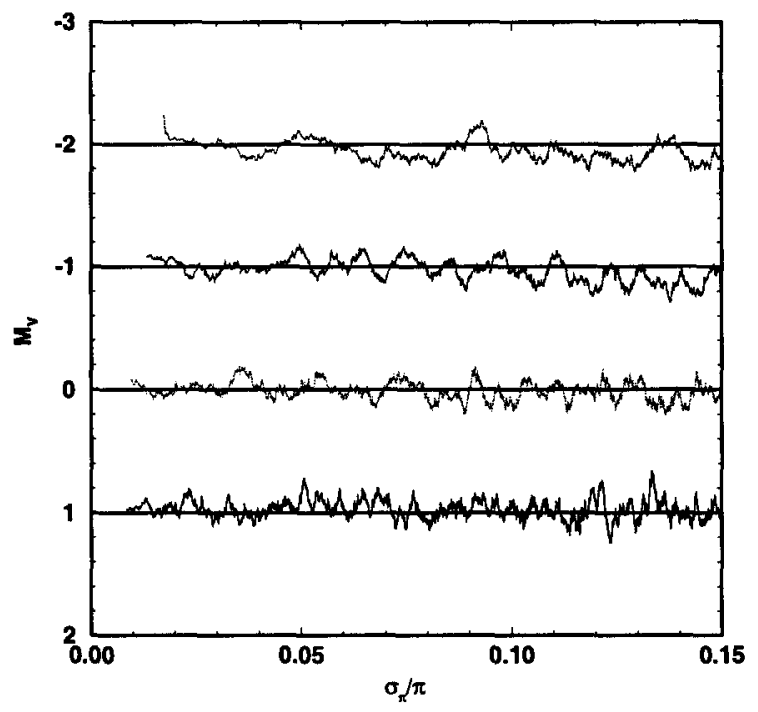

Figure 4. Same as Figure 3 for small $\sigma_{\pi} / \pi$

are not biased. But, as the regressions are weighted by the errors, stars having the smallest values of $\sigma_{\pi} / \pi$ preferentially determine the regressions computed from the entire samples of B and Be stars. Moreover, as can be seen in Figure 3, the biases can be positive or negative and thus can compensate one another. 


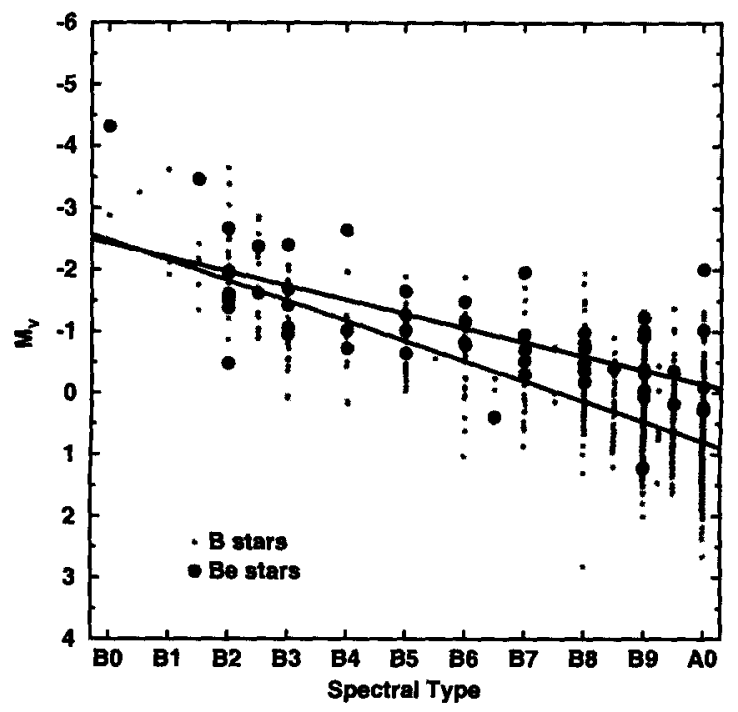

Figure 5. HR diagram of $\mathrm{B}$ and Be stars with $\sigma_{\pi} / \pi \leq 15 \%$. The lines indicate linear regressions weighted by the individual errors on the absolute magnitudes.

Figure 6 exhibits a comparison of the weighted mean magnitudes that we obtained for B stars (without emission) with absolute magnitudes given by Schmidt-Kaler (1982), and Zorec and Briot (1991) for the luminosity class V. For B stars later than B5, there is a good agreement with Zorec \& Briot (1991), but the sequence of Schmidt-Kaler (1982) is 0.3-0.5 magnitudes brighter. For spectral types between B2 and B5, the mean Hipparcos absolute magnitudes are systematically fainter by 0.5-1 magnitude than those of the other studies. For the earliest spectral types O9-B2, nothing can be said because of the lack of accurate data.

The mean values of absolute magnitudes of $B$ and Be stars for each spectral type are plotted on Figure 7. The error bars are the errors of the mean $\sigma_{M_{V}} / \sqrt{N}$.

The most interesting result shown by Figure 7 (and the regressions of Figure 5) is the general trend of Be stars to be brighter than B stars of the same spectral type for spectral types later than about B4, and the later the spectral type, the larger the over-luminosity which reaches 1 magnitude for A0 stars.

The mean values of $\sigma_{M_{V}}$ are plotted on Figure 8 and are 0.5-0.6 for B stars. The width of the Be sequence is not very well defined due to the small number statistics but it does not seem larger than the B sequence. This is quite surprising because emission characteristics of Be stars are very inhomogeneous. If we define the width of the sequences to be equal to $4 \sigma_{M_{V}}$ (i.e. including more than $95 \%$ of the stars in the case of a normal distribution), this width is about 2.5 magnitudes and is thus larger than the observed over-luminosity of Be stars. But the over-luminosity is statistically significant as can be seen by the size of the errors of the means in Figure 7. The regression lines, as well as the mean 


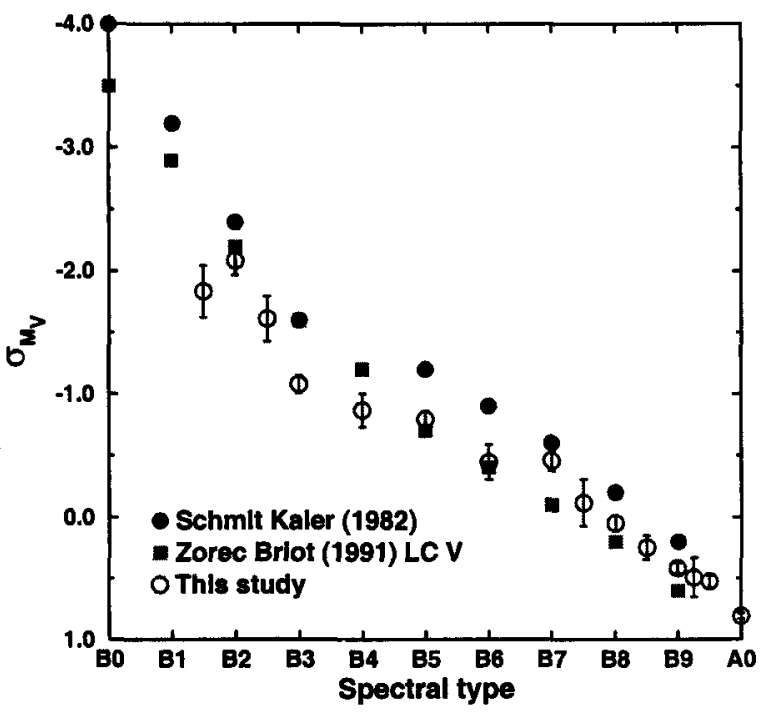

Figure 6. HR diagram - Absolute magnitude $M_{V}$ of B stars vs Spectral Type from the literature

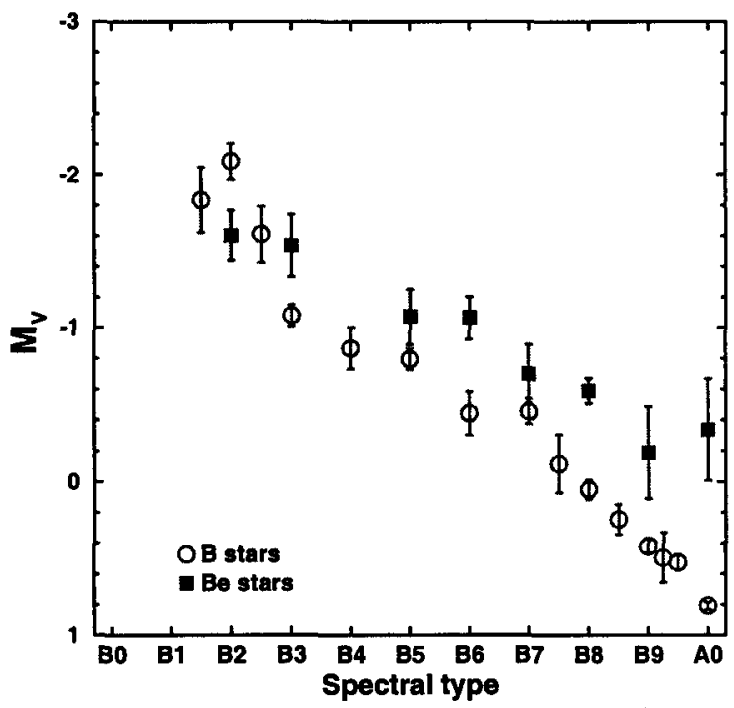

Figure 7. HR diagram - mean values of $M_{V}$ for $\sigma_{\pi} / \pi \leq 15 \%$

$M_{V}$, have been computed to exhibit the tendency of Be stars to be brighter than "normal" B stars. Any relation between the absolute magnitudes and the spectral types when the width of the sequences is so large should be considered with caution. 


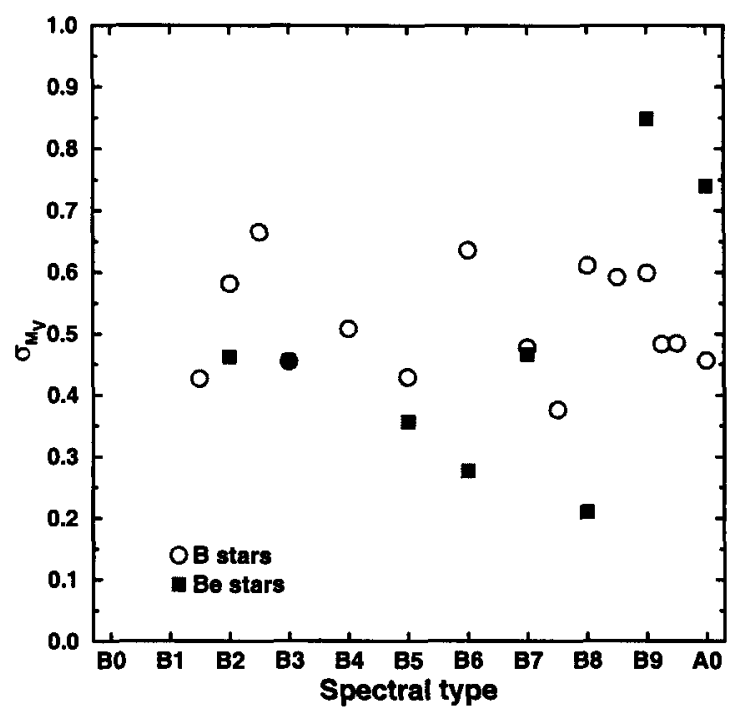

Figure 8. mean values of $\sigma_{M_{V}}$ for $\sigma_{\pi} / \pi \leq 15 \%$

\section{A possible physical interpretation of the over-luminosity of $\mathrm{Be}$ stars}

At first glance, the over-luminosity of late Be stars, compared to that of B stars without emission, may be surprising. The strength of the emission characteristics strongly decreases towards late B sub-spectral types so as the late Be stars do not appear very different from the B stars of the same spectral types. However, it is known that the mean rotational velocity of late Be stars is larger than that of early Be stars (see for example Briot 1986) and that the critical rotational velocity is smaller for stars with lower mass (Collins et al. 1991). Hence, late Be stars rotate near their critical velocities (Slettebak 1979).

Extensive calculations of energy distributions emitted by rigidly rotating B stars by Collins et al. (1991) show that their visible absolute magnitude $M_{V}$ and $B-V$ colour are modified by rotational effects. The corresponding displacements in the HR diagram are larger the closer to critical is the stellar rotation. The effect is also a function of the aspect angle $i$. Stars seen pole-on look brighter, although they almost preserve their non-rotational $B-V$ colour $\left(\Delta M_{V} \simeq 0.5 \mathrm{mag}\right.$. at critical rotation), while stars seen equator-on are reddened at almost constant luminosity. In any case, a given rotational sequence $\left(\Omega / \Omega_{c}=\right.$ const.) is above the corresponding luminosity class sequence of non-rotating stars. Hence, rotating stars look brighter and show cooler spectral types than expected for zero-rotating stars of the same mass. On the other hand, rotating stars do not evolve in the same way as non-rotating stars (Endal \& Sofia 1979 ) and the effects of the rotation-evolution interactions are stronger for late $\mathrm{B}$ masses where, on average, rotation is closer to critical. In particular, evolving rotating stars may become differential rotators and the degree of differential rotation is higher for more evolved stars (Zorec et al. 1987). The apparent 
displacements on the HR diagram are then also a function of the internal rotational law (Collins \& Smith 1985). So, at the moment, rotation seems to be the phenomenon which most likely produces the over-luminosity of late Be stars.

\section{Conclusion - Look at the future}

The results obtained from Hipparcos are quite new and interesting. However, some problems remain and new studies are desirable. This study is based on small number statistics and some biases remain. An example of a bias on the selection of the stars is given on the Figure 9 which shows the location of the studied stars in the sky. Southern B stars are much more numerous than northern ones for the simple reason that a survey catalogue of spectral types (the Michigan Spectral Survey, see Houk \& Smith-Moore 1988) is available in the southern sky; for the northern sky none are. The spectral type determinations of $\mathrm{B}$ and Be stars have to be improved and made in a homogeneous way.

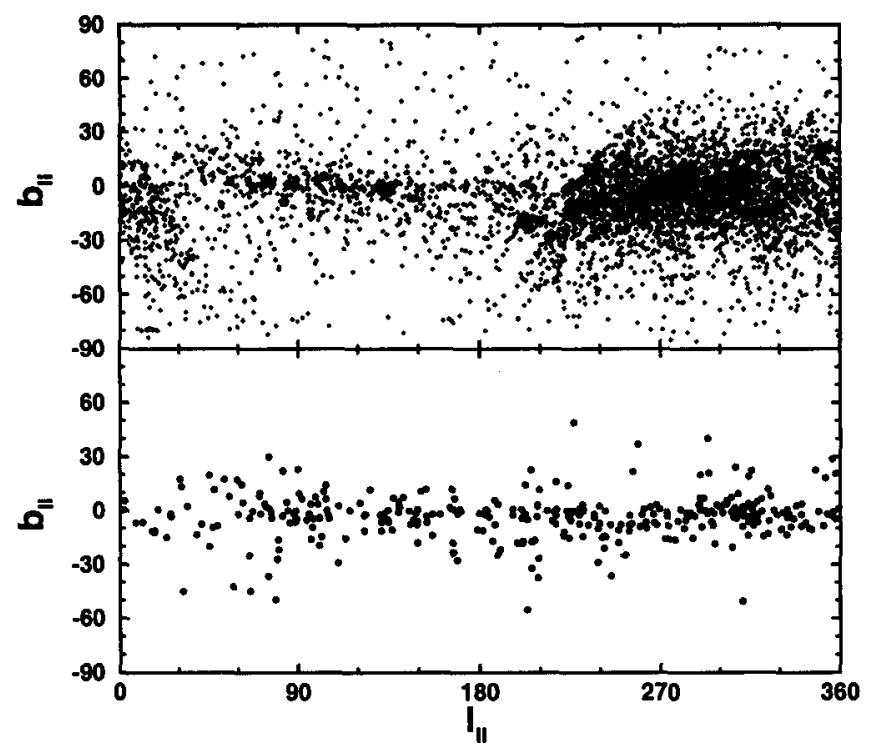

Figure 9. Distribution of B (top) and Be (bottom) stars over the sky

Another cause of error can be the undetected Be stars among B stars, especially among the faintest stars of the sample. If Be stars have been detected because they were the brightest among their spectral type, a very severe bias can result. A few other problems such as undetected binaries, evolutionary effect, etc. can of course bias the magnitudes as well.

Huge progress in the knowledge of $\mathrm{B}$ and $\mathrm{Be}$ stars will be made by exploiting the GAIA satellite which is a proposed cornerstone mission of the ESA $2000+$ program. Launch is planned around 2009. The scientific goals have been reviewed by Perryman et al. (1997). This mission will determine positions, proper motions, and parallaxes of about 35 millions objects down to about $\mathrm{V}=$ 15 with an accuracy better than 10 microarcsec, and of about 1 billion objects 
down to $\mathrm{V}=20$ with an accuracy better than 200 microarcsec. By comparison, the Hipparcos accuracy was about 1 milliarcsec with a limiting magnitude around 12. As a result, $1 \%$ of the Milky Way population will be observed (and a much larger fraction of $B$ stars). The satellite will also measure magnitudes in 4 broad photometric bands and 11 intermediate ones and will be equipped with a low resolution spectrometer allowing one to measure radial velocities and a few other stellar parameters.

Some advantages of GAIA for the study of B and Be stars are as follows:

- As one of the narrow band is centred on $H_{\alpha}$, GAIA will be able to separate emission line stars from the others and will thus provide secure samples of Be and B stars,

- Very accurate determinations of parallaxes and thus absolute magnitudes for a very large sample of $B$ and Be stars will be obtained. We will be able to determine whether Be stars in the galactic field, in galactic clusters and associations, and even in the various clusters of the Magellanic Clouds are the same population objects whatever the metallicity of the cluster,

- the distances of $B$ and Be stars will be determined very carefully. These intrinsically bright stars will be used as milestones for the study of the Milky Way. As young objects, they are yet very close to their birth place. In the galactic field, we will determine the location of $\mathrm{B}$ and Be stars with respect to the spiral arms. Just as Burki \& Maeder (1977) found a higher proportion of Be stars in the direction of galactic centre, and a lower one in the anticentre direction, we will study the distribution of $\mathrm{B}$ and $\mathrm{Be}$ stars in these special directions, and connect it to the formation process of rotating stars,

- the nonlinear motion of the photocentre of unresolved systems measured by GAIA will allow the detection of many Be binaries,

- accurate kinematical study of $\mathrm{B}$ and $\mathrm{Be}$ stars can provide information about their origin, and their age,

- five years of photometric monitoring will allow extensive studies of Be variability: microvariabity, mid-term and long-term variability.

In conclusion, among Hipparcos results for Be stars the over-luminosity of late type Be stars is the most striking result. This finding constrains models of rotating stars. The widths of both $\mathrm{Be}$ and $\mathrm{B}$ sequences have also to be interpreted, especially for B stars. Hipparcos results for Be stars can be expected to give yet more interesting results. In the future, the GAIA mission will immensely extend our knowledge of these intriguing stars.

Acknowledgments. It is a pleasure to thank J. Zorec and F. Arenou for their help and C. Turon for providing information about GAIA.

\section{References}

Arenou, F., Grenon, M., Gomez, A., 1992, A\&A, 258, 104

Arenou, F., Luri, X., 1999, in Harmonizing cosmic distance scales in a postHipparcos era, ed. D. Egret \& A. Heck, ASP conf. Series 167, p. 13 
Briot, D., 1986, A\&A, 163, 67

Briot, D., Hubert, A.M., Vakili, F., Floquet, M., Hubert, H., 1995, in Future Possibilities for Astrometry in Space, ESA SP-379, p. 87

Briot, D., Robichon, N., Hubert, A.M., 1997, in Hipparcos Venice'97, ESA-SP 402, p. 319

Burki, G., Maeder, A. 1977, A\&A, 57, 405

Collins, G.W., Smith, R.C., 1985, MNRAS, 213, 519

Collins, G.W., Truax, R.J., Cranmer, S.R., 1991, ApJS, 77, 541

Endal, A.S., Sofia, S., 1979, ApJ, 232, 531

ESA, 1997, The Hipparcos and Tycho Catalogues, SP-1200

Houk, N., Smith-Moore, A., 1988, Michigan catalogue of Two-Dimensional spectral types for the HD stars, Vol. 4: -26 to -12 degrees, Univ Michigan

Jaschek, M., Egret, D., 1982, Catalogue of Stellar Groups, SP. Publ. CDS 4, Obs. Strasbourg

Lutz, T.E., Kelker, D. H., 1973, PASP, 85, 573

Malmquist, K.G., 1936, Meddel. Stockholm Obs. 26

Merrill, P.W., 1933, PASP, 45, 198

Perryman M.A.C., Lindegren L., Turon C., 1997, in Hipparcos Venice'97, ESA SP-402, p. 743

Schmidt-Kaler, Th., 1982, Landolt-Börntein, New series, group VI, Vol. 2, Subvol. b, p.17

Slettebak, A., 1979, Space Sci.Rev., 23, 541

Slettebak, A., 1982, ApJS, 50, 55

Zorec, J., Briot, D., 1991, A\&A, 245, 150

Zorec, J., Divan, L., Mochkovitch, R., Garcia, A., 1987, IAU Coll. 92, p. 68

\section{Discussion}

D. Gies: If the Be stars are members of an older population, could you explain their over-luminosity by post-main sequence brightening?

D. Briot: Be stars may be members of an older population, but to avoid as possible this effect we only consider stars of luminosity class V and IV. Anyway, one should explain how this effect would be so more important for late Be stars. P. Harmanec: Cannot the spread in $M_{V}$ for absorption B stars be due many of them being short period binaries with similar components (a factor of $2.5 \log 2$ mag brighter) and some of them could be unrecognised Be stars?

D. Briot: The spread of the B star sequence can obviously be due to undetected binaries, but it can also be due to evolutionnary effects, wrong spectral types, rotation, chemical composition, etc., as it can be for the Be stars. As much as possible, Be stars were eliminated; yet some of them can remain. The effect due to (probable) unrecognized Be stars would be only significant for late type B stars, because the mean absolute magnitude of early Be stars is not different of that of early "normal" B stars.

P. Harmanec: What magnitude did you use for Be stars given the fact that they are light variables with $\Delta V$ range often ever $0.5 \mathrm{mag}$ ? 
D. Briot: We used magnitudes given in the Hipparcos catalogue, that is their average magnitudes.

P. Harmanec: A comment: If there is still chance to affect the technical specifications of the GAIA satellite, I should urge to remove the upper limit (5$8 \mathrm{mag}$ ) in the magnitude. There are many supergiants in the magnitude range from 3 to 6 mag for which Hipparcos parallaxes are not accurate enough. They need to be observed with a higher accuracy!

J. Bjorkman: You mentioned that late type Be stars may be more rapidly rotating than early type Be stars. Could gravity darkening effects cause a systematic spectral type shift (toward later ST) that could explain their higher magnitudes?

D. Briot: Late type Be stars rotate on average more rapidly than early type stars, but above all they rotate near their critical velocity. Models show that in this case gravity darkening effects are significat and most effective in carrying systematic spectral type shifts for stars seen equator-on. Up to now, we could not find a correlation between over-luminosity and inclination of late-type $\mathrm{Be}$ stars. The luminosity of pole-on stars rotating near their critical velocity is enhanced. Several effects act together on the luminosity of a rotating star.

E. Alfaro: How many stars in your sample are in open clusters? What about the comparison of absolute magnitudes derived from both methods?

D. Briot: Only a few Be stars are in open clusters. During the Hipparcos mission, the number of objects observed per square degree was limited to 5 . So we could not make a comparison because of the scarcity of the sample of $\mathrm{Be}$ stars in open clusters

E. Alfaro: I have a concern about the use of the Arenou et al. (1992) model for correcting extinction:

- this model is only valid for distances to the Sun of the order of $500 \mathrm{pc}$

- It does not take into account local variations of extinction which are typically present surrounding Be stars.

D. Briot: According to F. Arenou, his model is valid up to about $1 \mathrm{kpc}$. As we have chosen stars with $\sigma_{\pi} / \pi \leq 15 \%$ and as $\sigma_{\pi}$ is never smaller than 0.5 mas, it insures us that the selected stars are well inside this volume. Concerning the second point, any extinction not properly taken into account would even increase the absolute magnitudes of Be stars further. 